\title{
NULL-CONTROLLABILITY OF SOME SYSTEMS OF PARABOLIC TYPE BY ONE CONTROL FORCE
}

\author{
Farid Ammar Khodja ${ }^{1}$, Assia Benabdallah ${ }^{2}$, CÉdric Dupaix $^{1}$ and Ilya Kostin $^{3}$
}

\begin{abstract}
We study the null controllability by one control force of some linear systems of parabolic type. We give sufficient conditions for the null controllability property to be true and, in an abstract setting, we prove that it is not always possible to control.
\end{abstract}

Mathematics Subject Classification. 93B05, 93C20, 93C25, 35K90.

Received March 3, 2003. Revised May 17 and October 14, 2004.

\section{INTRODUCTION}

Null-controllability of linear and semilinear parabolic equations has been extensively studied these last ten years. In their work, Lebeau and Robbiano [6] considered the heat equation in bounded domains $\Omega \subset R^{n}$ and for positive times $T$

$$
\begin{array}{rlrl}
u_{t} & =\Delta u+f \chi_{\omega} & & \text { in } Q_{T}=(0, T) \times \Omega \\
u & =0 & & \text { on } \Sigma_{T}=(0, T) \times \partial \Omega \\
u(0, .) & =u_{0} \in L^{2}(\Omega) &
\end{array}
$$

where $\omega$ is an open set which satisfies $\bar{\omega} \subset \Omega$. They proved its null-controllability by a localized (in space) control: for any $u_{0} \in L^{2}(\Omega)$, there exists $f \in L^{2}\left(Q_{T}\right)$ such that the associated solution of (1) satisfies $u(T,.) \equiv 0$ in $\Omega$. The main tool to establish such a result is a Carleman estimate. Fursikov and Imanuvilov [7] proved the same result for the problem:

$$
\begin{array}{rlr}
L u & =f \chi_{\omega} \quad & \text { in } Q_{T} \\
u & =0 \quad \text { on } \Sigma_{T} \\
u(0, .) & =u_{0} \in L^{2}(\Omega)
\end{array}
$$

\footnotetext{
Keywords and phrases. Control, parabolic systems.

${ }^{1}$ Université de Franche-Comté Département de Mathématiques CNRS-UMR 662316 Route de Gray, 25030 Besançon Cedex, France; ammar@math.univ-fcomte.fr; dupaix@math.univ-fcomte.fr

${ }^{2}$ Université de Provence, CMI - LATP Technopôle Château-Gombert, 39 rue F. Joliot Curie, 13453 Marseille Cedex 13, France; assia@cmi.univ-mrs.fr

${ }^{3}$ Université de Saint-Etienne, Équipe d'Analyse Numérique, 23 rue Paul MICHELON, 42023 Saint-Etienne Cedex 02, France; kostin@free.fr 
where $L$ is a general linear second order parabolic operator. Again, the main tool is a global Carleman estimate satisfied by the solutions of (2). This estimate allowed these authors to prove the null-controllability (controllability to the trajectories) of semilinear equations:

$$
\begin{aligned}
L u & =h(u)+f \chi_{\omega} & & \text { in } Q_{T} \\
u & =0 & & \text { on } \Sigma_{T} \\
u(0, .) & =u_{0} \in L^{2}(\Omega) & &
\end{aligned}
$$

when $h$ is a globally Lipschitz-continuous function. Fernandez-Cara and Zuazua [8] and Barbu [4] generalized this result to some superlinearities using a fixed point method and a precise expression of the constants appearing in the global Carleman estimate.

In contrast, very few results are published for linear or semilinear parabolic systems. Anita and Barbu [3], for a reaction-diffusion system, and Barbu [5] for the phase field system, proved local exact controllability results by two localized (in space) control forces. In a recent paper [1], the authors give local exact controllability and exact controllability results for phase field systems by only one localized control force. All these papers pass first through the study of the controllability of a "linearized" version of the system and use some Carleman estimate in order to apply a fixed point theorem.

As it is by now well-known, the controllability result of this linearized system amounts to prove an observability estimate for its adjoint system. This part is in a way the corner-stone for the proof of the controllability of the initial semilinear problem when using a fixed point method. In particular if a control problem by only one force is considered, it is to obtain this estimate that the coupling operator between the equations of the system plays role. So in view of these facts, a natural question arises: is it always possible to control coupled linear parabolic systems by a reduced number of control forces. It is the aim of this paper to give some partial answers to this question.

To address this question, this article is organized as follows.

We first consider in Section 2 a linear system which naturally appears when dealing with the controllability of some nonlinear reaction-diffusion systems. In this case, the observability estimate is related to the existence of a definite, non-negative quadratic form $\Phi$, which is introduced in the proof of Lemma 1 . This $\Phi$ takes into account the way in which the equations of the system are coupled and it leads to the desired observability estimate. The goal of this section is to illustrate, in a simple case, the main ideas contained in the proof of such a result.

In the light of Section 2, one may ask if it is possible to adapt this approach to general abstract linear systems of parabolic type (at least to obtain some sufficient condition for their controllability). This is the goal of Section 3 where it turns out that the observability estimate is actually related to the existence of such a $\Phi$ which is itself related to the existence of a multiplier $M$ as shown in Theorem 2.1. Note that this relation between $\Phi$ and $M$ was hidden in Section 2 since in this case $M$ was equal to the identity. Finally, to conclude this third section we exhibit some examples of linear "parabolic-like" systems showing that it is not always possible to control such coupled systems by a reduced number of control forces. In this case the non-controllability is related to the compactness of the coupling operator (this operator being in some sense "too compact"). Note that the operators in these examples are not of partial differential type and thus do not seem to be physically relevant.

In order to illustrate the relevance of the assumption concerning the existence of a multiplier $M$ in Theorem 2.1, we present in Section 4 an example of linear parabolic system for which it is not possible to take the identity for $M$. We show how the abstract theorem of Section 3 can be used for this example to deduce the controllability of the system by a single (but non-localized) control force. Finally, using this first result together with suitable Carleman estimates we obtain the controllability of the system by a single localized control force. 


\section{An eXAmple: Reaction-Diffusion systems}

We consider a general reaction-diffusion system which arises in mathematical biology:

$$
\left\{\begin{array}{l}
\psi_{t}=\Delta \psi+h(\psi, w)+w \text { on } \Omega \times \mathbb{R}^{+} \\
w_{t}=\Delta w+g(\psi, w)+f \text { on } \Omega \times \mathbb{R}^{+} \\
\left.\psi\right|_{\delta \Omega}=\left.w\right|_{\delta \Omega}=0 \text { in } \mathbb{R}^{+} \\
\psi(x, 0)=\psi_{0}, w(x, 0)=w_{0}
\end{array}\right.
$$

where $\Omega$ is a bounded domain of $\mathbb{R}^{n}$ with smooth boundary, $h, g$ smooth functions and $f$ a source term. Let $T>0, f^{*}$ in $L^{2}\left(Q_{T}\right)$ (with $\left.Q_{T}=\Omega \times\right] 0, T\left[\right.$ ), and $\left(\psi_{0}^{*}, w_{0}^{*}\right) \in L^{2}(\Omega)^{2}$. Suppose that there exists a $\left(\psi^{*}, w^{*}\right)$ satisfying (4) in $\left.\left.(C(] 0, T] \times L^{2}(\Omega)\right)\right)^{2}$ with $(\psi(0), w(0))=\left(\psi_{0}^{*}, w_{0}^{*}\right)$ and consider the controllability question of finding a function $f \in L^{2}\left(Q_{T}\right)$ such that the corresponding solution pair $(\psi, w)$ of $(4)$ satisfies $\psi(T)=\psi^{*}(T)$ and $w(T)=w^{*}(T)$. Note that this is still an open question with a single control force (even for a non-localized control) which will be address in a future work [2]. To our knowledge, the only result in this direction is the one of Anita and Barbu [3] for a localized control acting on both equations. The sketch of the proof of such a result, is to set first

$$
\begin{aligned}
\psi & =\bar{\psi}-\psi^{*} \\
w & =\bar{w}-w^{*}
\end{aligned}
$$

where $(\bar{\psi}, \bar{w})$ satisfies $(4)$, to get:

$$
\left\{\begin{array}{l}
\psi_{t}=\Delta \psi+\left(h(\bar{\psi}, \bar{w})-h\left(\psi^{*}, w^{*}\right)\right)+w \text { on } \Omega \times \mathbb{R}^{+} \\
w_{t}=\Delta w+\left(g(\bar{\psi}, \bar{w})-g\left(\psi^{*}, w^{*}\right)\right)+f-f^{*} \text { on } \Omega \times \mathbb{R}^{+} \\
\left.\psi\right|_{\delta \Omega}=\left.w\right|_{\delta \Omega}=0 \text { in } \mathbb{R}^{+} \\
\psi(x, 0)=\psi_{0}-\psi_{0}^{*}, w(x, 0)=w_{0}-w_{0}^{*} .
\end{array}\right.
$$

The main task is now to prove the controllability of a linearization of this system with suitable estimates on the control (with the help of Carleman estimates if the control is localized) to be able to use a fixed point argument. As mentioned in the introduction, the question under interest here is the study of the controllability of this linearized system. This system takes here the following form:

$$
\left\{\begin{array}{l}
\psi_{t}=\Delta \psi+a \psi+b w \text { on } \Omega \times \mathbb{R}^{+} \\
w_{t}=\Delta w+d \psi+c w+f \text { on } \Omega \times \mathbb{R}^{+} \\
\left.\psi\right|_{\delta \Omega}=\left.w\right|_{\delta \Omega}=0 \text { in } \mathbb{R}^{+} \\
\psi(x, 0)=\psi_{0}, w(x, 0)=w_{0}
\end{array}\right.
$$

We assume $a, b, c, d \in L^{\infty}\left(Q_{T}\right)$ and $f \in L^{2}\left(Q_{T}\right)$.

The rest of this section is now devoted to the study of the controllability of (5). To begin with we recall for completeness why this controllability property is actually equivalent to the so called observability estimate. Then we prove in Lemma 1.2 this estimate.

Existence and uniqueness for (5) follow from classical results on parabolic systems (see [9]). System (5) can be written in the following abstract form:

$$
Y_{t}=A(t) Y+B f
$$

with

$$
\begin{gathered}
A(t)=\left(\begin{array}{cc}
\Delta+a I & b I \\
d I & \Delta+c I
\end{array}\right), \\
Y=\left(\begin{array}{c}
\psi \\
w
\end{array}\right), \quad B=\left(\begin{array}{l}
0 \\
I
\end{array}\right) .
\end{gathered}
$$


One gets that for all initial data $\left(\psi_{0}, w_{0}\right) \in H=L^{2}(\Omega) \times L^{2}(\Omega)$ and all $f$ in $L^{2}\left(Q_{T}\right)$, there exists a unique solution $(\psi, w) \in C([0, T] ; H)$.

Our aim is to prove that for all initial data $Y_{0}=\left(\begin{array}{c}\psi_{0} \\ w_{0}\end{array}\right) \in H$, one can find $f \in L^{2}\left(Q_{T}\right)$ such that the corresponding solution of (5) satisfies

$$
Y(T)=0
$$

As in [13], for any $t>0$, we define the operators $S_{t}$ which associates with an initial data $Y_{0} \in H$ the solution of the homogeneous system $\left((5)(f=0)\right.$ at time $t$ and $L_{t}$ which associates with $f \in L^{2}\left(Q_{T}\right)$ the solution at time $t$ of (5) corresponding to null initial data. Then, the controllability property is equivalent to the existence, for all initial data in $H$, of $f \in L^{2}(\Omega)$ such that

$$
S_{T} Y_{0}+L_{T} f=0
$$

which is equivalent to

$$
R\left(S_{T}\right) \subset R\left(L_{T}\right) .
$$

This last inclusion holds if and only if (see for instance [13], Th. 2.2, p. 208) there exists $C_{T}>0$ such that

$$
\left\|S_{T}^{*}\left(Y_{0}\right)\right\|_{H} \leq C_{T}\left\|L_{T}^{*} Y_{0}\right\|_{L^{2}(0, T ; H)} \quad \forall Y_{0} \in H,
$$

where, for any bounded operator $M, M^{*}$ denotes the adjoint operator. Since $\left(L_{T}^{*} h\right)(t)=B^{*} S_{T-t}^{*} h$ for any $h \in H$ and if $A^{*}(t)$ denotes the adjoint operator in $H$ of $A(t)$, this last inequality can be written as

$$
|\phi(T)|^{2}+|u(T)|^{2} \leq C_{T} \int_{0}^{T} \int_{\Omega}|u(t, x)|^{2} \mathrm{~d} x \mathrm{~d} t,
$$

where |.| stands for the $L^{2}$-norm and $(\phi, u)$ is the solution of the adjoint problem:

$$
\left\{\begin{array}{l}
\phi_{t}=\Delta \phi+a \phi+d u \text { on } \Omega \times \mathbb{R}^{+} \\
u_{t}=\Delta u+c u+b \phi \text { on } \Omega \times \mathbb{R}^{+} \\
\left.\phi\right|_{\delta \Omega}=\left.u\right|_{\delta \Omega}=0 \text { in } \mathbb{R}^{+} \\
\phi(x, 0)=\phi_{0}, u(x, 0)=u_{0} .
\end{array}\right.
$$

Remark 1.1. We insist again here on the fact that the purpose of this article is to study the way to prove the observability estimate in the case of a reduced number of control forces. In particular we are not interested at this level by the localization in space of the control. However let us mention that if $f=g \chi_{\omega}$ in (5) then the corresponding observability estimate (namely with $\omega$ instead of $\Omega$ in the right-hand-side of (9)) can be deduced by using some suitable Carleman estimate (see [2]).

Let us show the observability inequality (9) for solutions of (10).

Lemma 1.2. For $T>0$, assume that $b \geq \delta>0$ a.e in $Q_{T}$. Then there exists a positive constant $C_{T}$ such that for all initial data $\left(\phi_{0}, u_{0}\right) \in L^{2}(\Omega) \times L^{2}(\Omega)$ the corresponding solution of (10) satisfies (9).

Proof. (9) is an observability estimate. It shows the possibility of recovering the total $L^{2}$-norm at time $T$ by observing only the $L^{2}$-norm of $u$ from $t=0$ to $t=T$. The proof of this Lemma is based on the introduction of a suitable functional itself based on suitable multipliers. Let us introduce, for any solution $(\phi, u)$ of $(10)$, the functional:

$$
\Phi(\phi, u)=t^{4}|\phi|^{2}+\lambda t^{2}|u|^{2}-\mu t^{3}(\phi, u),
$$

where $\lambda, \mu$ are positive constants witch will be chosen later. 
One has

$$
\begin{aligned}
\frac{\mathrm{d}}{\mathrm{d} t}\left(t^{4}|\phi|^{2}\right)= & 4 t^{3}|\phi|^{2}+2 t^{4}\left(\phi, \phi_{t}\right) \\
\leq & 4 t^{3}|\phi|^{2}-2 t^{4}|\nabla \phi|^{2}+2 t^{4}\|a\|_{L^{\infty}\left(Q_{T}\right)}|\phi|^{2} \\
& +2 t^{4}\|d\|_{L^{\infty}\left(Q_{T}\right)}|(\phi, u)| \\
\frac{\mathrm{d}}{\mathrm{d} t}\left(t^{2}|u|^{2}\right)= & 2 t|u|^{2}+2 t^{2}\left(u, u_{t}\right) \\
\leq & 2 t|u|^{2}-2 t^{2}|\nabla u|^{2}+2 t^{2}\|c\|_{L^{\infty}\left(Q_{T}\right)}|u|^{2} \\
& +2 t^{2}\|b\|_{L^{\infty}\left(Q_{T}\right)}|(\phi, u)|,
\end{aligned}
$$

and

$$
\begin{aligned}
-\frac{\mathrm{d}}{\mathrm{d} t} t^{3}(\phi, u) \leq & 3 t^{2}|(\phi, u)| \\
& +t^{3}\left\{2|(\nabla \phi, \nabla u)|+\left(\|a\|_{L^{\infty}\left(Q_{T}\right)}+\|c\|_{L^{\infty}\left(Q_{T}\right)}\right)|(\phi, u)|\right\} \\
& -t^{3}(b \phi, \phi)+t^{3}\|d\|_{L^{\infty}\left(Q_{T}\right)}|u|^{2} .
\end{aligned}
$$

Thus, differentiating $\Phi$ with respect to time, we obtain:

$$
\frac{\mathrm{d}}{\mathrm{d} t} \Phi(u, v) \leq I_{1}+I_{2}+I_{3}
$$

where we have introduced the following notations

$$
\begin{aligned}
I_{1}= & 4 t^{3}|\phi|^{2}-2 t^{4}|\nabla \phi|^{2}+2 t^{4}\|a\|_{L^{\infty}\left(Q_{T}\right)}|\phi|^{2}-\mu t^{3}(b \phi, \phi), \\
I_{2}= & \left(2\|d\|_{L^{\infty}\left(Q_{T}\right)} t^{4}+\mu \quad\|(a, c)\|_{L^{\infty}\left(Q_{T}\right)} t^{3}+\left(2 \lambda\|b\|_{L^{\infty}\left(Q_{T}\right)}+3 \mu\right) t^{2}\right)|(\phi, u)| \\
& +2 \mu|(\nabla \phi, \nabla u)| t^{3},
\end{aligned}
$$

where $\|(a, c)\|_{L^{\infty}\left(Q_{T}\right)}=\|a\|_{L^{\infty}\left(Q_{T}\right)}+\|c\|_{L^{\infty}\left(Q_{T}\right)}$.

$$
I_{3}=2 \lambda t|u|^{2}-2 \lambda t^{2}|\nabla u|^{2}+2 \lambda t^{2}\|c\|_{L^{\infty}\left(Q_{T}\right)}|u|^{2}+\mu t^{3}\|d\|_{L^{\infty}\left(Q_{T}\right)}|u|^{2} .
$$

Now we estimate each of the three quantities $I_{1}, I_{2}$ and $I_{3}$.

- Estimate of $I_{1}$.

Recall that we have assumed that $b \geq \delta>0$ a.e. in $Q_{T}$. Therefore, choosing

$$
\mu=\frac{8+4 T\|a\|_{L^{\infty}\left(Q_{T}\right)}}{\delta}
$$

leads to

$$
I_{1} \leq-2\left(2+T\|a\|_{L^{\infty}\left(Q_{T}\right)}\right) t^{3}|\phi|^{2}-2 t^{4}|\nabla \phi|^{2}
$$


- Estimate of $I_{2}$.

Using Cauchy-Schwarz's and Young's inequalities, we have:

$$
\begin{aligned}
I_{2} \leq & \left(2+T\|a\|_{L^{\infty}\left(Q_{T}\right)}\right) t^{3}|\phi|^{2}+2 t^{4}|\nabla \phi|^{2}+\frac{\mu^{2}}{4} t^{2}|\nabla u|^{2} \\
& +\left[2\|d\|_{L^{\infty}\left(Q_{T}\right)} t^{2}+\mu\|(a, c)\|_{L^{\infty}\left(Q_{T}\right)} t\right. \\
& \left.+2 \lambda\|b\|_{L^{\infty}\left(Q_{T}\right)}+3 \mu\right]^{2} \frac{t|u|^{2}}{\left(2+T\|a\|_{L^{\infty}\left(Q_{T}\right)}\right)^{2}} .
\end{aligned}
$$

- Estimate of $I_{3}$.

$$
I_{3} \leq P_{1}(t)|u|^{2}-2 \lambda t^{2}|\nabla u|^{2}
$$

where

$$
P_{1}(t)=\mu\|d\|_{L^{\infty}\left(Q_{T}\right)} t^{3}+2 \lambda\|c\|_{L^{\infty}\left(Q_{T}\right)} t^{2}+2 \lambda t
$$

In view of these last estimates, choosing $\lambda \geq \frac{\mu^{2}}{8}$, one gets

$$
\frac{\mathrm{d}}{\mathrm{d} t} \Phi(u, v) \leq P(t)|u|^{2}
$$

with

$$
\begin{aligned}
P(t)= & P_{1}(t)+\frac{t}{\left(2+T\|a\|_{L^{\infty}\left(Q_{T}\right)}\right)^{2}}\left[2\|d\|_{L^{\infty}\left(Q_{T}\right)} t^{2}\right. \\
& \left.+\mu\left(\|a\|_{L^{\infty}\left(Q_{T}\right)}+\|c\|_{L^{\infty}\left(Q_{T}\right)}\right) t+2 \lambda\|b\|_{L^{\infty}\left(Q_{T}\right)}+3 \mu^{2}\right]^{2} .
\end{aligned}
$$

To end the proof, it remains to show that $\Phi(u, v)$ is a quadratic definite form in $L^{2}(\Omega)$ for $t=T$. This is a direct consequence of the definition of $\Phi$, with $\lambda=\mu^{2}$. Actually, one has:

$$
\Phi(u, v)(T) \geq\left(\frac{1}{2} T^{2}+\frac{1}{2} \lambda-\frac{1}{2} \sqrt{\left(T^{4}-\lambda T^{2}+\lambda^{2}\right)}\right) T^{2}\left(|\phi(T)|^{2}+|u(T)|^{2}\right) .
$$

Therefore, all solutions of (10) satisfy (9) with

$$
C_{T}=\frac{\|P\|_{L^{\infty}(O, T)}}{\left(\frac{1}{2} T^{2}+\frac{1}{2} \lambda-\frac{1}{2} \sqrt{\left(T^{4}-\lambda T^{2}+\lambda^{2}\right)}\right) T^{2}} \approx \frac{C}{T^{3}},
$$

since $\|P\|_{L^{\infty}(O, T)} \underset{0}{\sim} c_{1} T$ and $\frac{1}{2}\left(T^{2}+\lambda-\sqrt{\left(T^{4}-\lambda T^{2}+\lambda^{2}\right)}\right) T^{2} \underset{0}{\sim} c_{2} T$ where $C, c_{1}, c_{2}$ are positive constants independent of $T$.

Remark 1.3. The question of the optimality of the minimal norm steering control as $T \downarrow 0$ is by now wellknown. Let's briefly recall some background connected to this question. Consider an abstract and general 
control problem:

$$
\begin{aligned}
Y_{t} & =A Y+B u, \\
Y(0) & =Y_{0} \in H .
\end{aligned}
$$

where $(A, D(A))$ is an unbounded operator in a Hilbert space $H$ and $B$, the control operator, is defined from the control Hilbert space $X$ in $H$. If the null controllability property holds for this abstract system, then for arbitrary $T>0$ and $Y_{0} \in H$, there exists $u \in L^{2}((0, T) ; X)$ such that the corresponding solution satisfies $Y(T)=0$. Then one can look for the control $u$ which minimizes $\|u\|_{L^{2}(0, T ; X)}$. Let us denote it by $u_{T}$ and let the minimal energy function $E_{\min }(T)$ defined by

$$
E_{\min }(T):=\sup _{\left\|Y_{0}\right\|=1}\left\|u_{T}\left(Y_{0}\right)\right\|_{L^{2}(0, T ; X)} .
$$

When $\operatorname{dim} H<\infty$, it is well known that $E_{\min }(T)$ is exactly the best constant $\sqrt{C_{T}}$ of the observability inequality and it has been proved (see $[11,12]$ ) that $\sqrt{C_{T}} \sim_{0} T^{-k-\frac{1}{2}}$ where $k$ is the smallest integer such that

$$
\left\{\begin{array}{c}
A \in M_{n}(\mathbb{R}) \text { et } B \in M_{n, m}(\mathbb{R}) \\
r g\left[B, A B, \ldots, A^{k} B\right]=n .
\end{array}\right.
$$

If we consider the correspondence between our system and a finite dimensional one with:

$$
A=\left(\begin{array}{cc}
-\lambda+a & d \\
b & -\lambda+c
\end{array}\right), B=\left(\begin{array}{l}
0 \\
1
\end{array}\right), d \neq 0
$$

then a simple computation gives $k=1$ and then $T^{-k-\frac{1}{2}}=T^{-\frac{3}{2}}$. Therefore it is interesting to notice that our computations leads to the same behavior near $T=0$ since we have obtained $\sqrt{C_{T}} \sim{ }_{0} C T^{-\frac{3}{2}}$.

\section{Null-controllability for general systems}

\subsection{Sufficient conditions}

This section is devoted to the question of null-controllability for some general systems of parabolic type when using control forces acting on a single equation of the system.

More precisely, let $\mathcal{U}$ and $\mathcal{V}$ be two real Hilbert spaces with scalar-product $(,)_{\mathcal{U}}$ and $(,)_{\mathcal{V}}$ respectively, and let ||$_{\mathcal{U}}$ and ||$_{\mathcal{V}}$ denote the corresponding norms. Let $A$ and $C$ be linear self-adjoint operators with the domains $\mathcal{D}(A) \subset \mathcal{U}$ and $\mathcal{D}(C) \subset \mathcal{V}$, respectively. We assume that these operators are positive definite. We also introduce two linear, possibly unbounded, densely defined operators $B: \mathcal{D}(B) \subset \mathcal{V} \rightarrow \mathcal{U}$ and $D: \mathcal{D}(D) \subset \mathcal{U} \rightarrow \mathcal{V}$. Assume also that the sets

$$
\mathcal{D}(A) \cap \mathcal{D}(D) \cap \mathcal{D}\left(B^{*}\right), \quad \mathcal{D}(C) \cap \mathcal{D}(B) \cap \mathcal{D}\left(D^{*}\right)
$$

are dense in $\mathcal{U}$ and $\mathcal{V}$ so that the matrix operators

$$
\mathcal{A}=\left(\begin{array}{cc}
A^{2} & -D^{*} \\
-B^{*} & C^{2}
\end{array}\right), \quad \hat{\mathcal{A}}=\left(\begin{array}{cc}
A^{2} & -B \\
-D & C^{2}
\end{array}\right)
$$

be defined on a common dense set $\mathcal{F} \subset \mathcal{U} \times \mathcal{V}$. Finally, suppose that for any $(u, v) \in \mathcal{U} \times \mathcal{V}$ the inequality

$$
(B v, u)_{\mathcal{U}}+(D u, v)_{\mathcal{V}} \leq|A u|_{\mathcal{U}}^{2}+|C u|_{\mathcal{V}}^{2}
$$


holds. Thus $\mathcal{A}$ is a densely defined dissipative operator. It is therefore closable [10], p. 15, Theorem 4.5. Let us retain the notation $\mathcal{A}$ for its closure. The domain of the adjoint operator $\mathcal{A}^{*}$ contains the dense set $\mathcal{F}$ and thus $\mathcal{A}^{*}$ is also dissipative. Therefore both $\mathcal{A}$ and $\mathcal{A}^{*}$ generate $C_{0}$ semigroups of contractions [10], p. 15, Corollary 4.4 .

This allows us to consider the following initial value problem

$$
\begin{cases}\psi_{t}=-A^{2} \psi+D^{*} w & \mathcal{U} \\ w_{t}=B^{*} \psi-C^{2} w+f & \mathcal{V} \\ \psi(0)=\psi_{0}, w(0)=w_{0} & \end{cases}
$$

where $f \in L^{2}\left(\mathbb{R}^{+}, \mathcal{V}\right)$ is the control force.

The aim of this section is to find conditions under which system (12) is $L^{2}$-null-controllable at any time $T>0$, i.e., for any time $T>0$ and any initial data $Y_{0}=\left(\psi_{0}, w_{0}\right) \in \mathcal{U} \times \mathcal{V}$ there exists a control force $f \in L^{2}\left(\mathbb{R}^{+}, \mathcal{V}\right)$ for which the corresponding solution $(\psi, w)$ of $(12)$ vanishes at time $T$. It is by now classical (see for instance [13], Th. 2.6, p. 213) that, if we denote by $R: \mathcal{V} \rightarrow \mathcal{U} \times \mathcal{V}$ the operator defined by $R f=\left(\begin{array}{c}0 \\ f\end{array}\right)$, this property is equivalent to the existence of a constant $c_{T}>0$ such that for all $\left(\psi_{0}, w_{0}\right) \in \mathcal{U} \times \mathcal{V}$

$$
\left\|\mathrm{e}^{\mathcal{A}^{*} T} Y_{0}\right\|^{2} \leq c_{T} \int_{0}^{T}\left\|R^{*} \mathrm{e}^{\mathcal{A}^{*} t} Y_{0}\right\|^{2} \mathrm{~d} t
$$

which reads

$$
|u(T)|_{\mathcal{U}}^{2}+|v(T)|_{\mathcal{V}}^{2} \leq c_{T} \int_{0}^{T}|v(t)|_{\mathcal{V}}^{2} \mathrm{~d} t
$$

for the solution $(u, v)$ of the adjoint system,

$$
\left\{\begin{array}{l}
u_{t}=-A^{2} u+B v \\
v_{t}=D u-C^{2} v \\
u(0)=u_{0}, \quad v(0)=v_{0}
\end{array}\right.
$$

Throughout this section we agree to denote by $c$ a constant depending only on the operators $A, B, C$, and $D$.

The main result of this section reads as follows:

Theorem 2.1. Let $\left(u_{0}, v_{0}\right) \in D(\mathcal{A})$. Assume that there exists a linear (maybe unbounded but not necessarily closed) operator $M: \mathcal{D}(M) \subset \mathcal{V} \rightarrow \mathcal{U}$ such that for some $p \in \mathbb{R}$ the following inequalities are defined and hold for any $(u, v) \in D(\mathcal{A})$ :

$$
\begin{aligned}
-(B v, M v)_{\mathcal{U}} & \leq c|C v|_{\mathcal{V}}^{2} \\
\left|M^{*} u\right|_{\mathcal{V}}^{2} & \leq c\left|A^{-p} u\right|_{\mathcal{U}}^{2} \\
\left|A^{-1-p} B v\right|_{\mathcal{U}}^{2} & \leq c|C v|_{\mathcal{V}}^{2} \\
(M D u, u)_{\mathcal{U}} & \geq c\left|A^{-p} u\right|_{\mathcal{U}}^{2} \\
\left|A^{1+p} M v\right|_{\mathcal{U}}^{2} & \leq c|C v|_{\mathcal{V}}^{2} \\
\left|A^{-1+p} M C^{2} v\right|_{\mathcal{U}}^{2} & \leq c|C v|_{\mathcal{V}}^{2} .
\end{aligned}
$$


Assume also that there exists a positive constant $\gamma$ such that for all $\varepsilon>0$,

$$
\begin{aligned}
\left|A^{-1+p} D^{*} v\right|_{\mathcal{U}}^{2} & \leq \varepsilon|C v|_{\mathcal{V}}^{2}+\frac{c}{\varepsilon^{\gamma}}|v|_{\mathcal{V}}^{2} \\
\left|A^{-1+p} M v\right|_{\mathcal{U}}^{2} & \leq \varepsilon|C v|_{\mathcal{V}}^{2}+\frac{c}{\varepsilon^{\gamma}}|v|_{\mathcal{V}}^{2}
\end{aligned}
$$

for any $(u, v) \in \mathcal{F}$. Then for any $T>0$ the solution of problem (14) satisfies the estimate

$$
\left|A^{-p} u(T)\right|_{\mathcal{U}}^{2}+|v(T)|_{\mathcal{V}}^{2} \leq c T^{-2 \gamma-4} \int_{0}^{T}|v(t)|_{\mathcal{V}}^{2} \mathrm{~d} t
$$

If, in addition, there exists a constant $q>0$ such that the estimate

$$
|u(T)|_{\mathcal{U}}^{2}+|v(T)|_{\mathcal{V}}^{2} \leq c\left(1+T^{-q}\right)\left(\left|A^{-p} u_{0}\right|_{\mathcal{U}}^{2}+\left|v_{0}\right|_{\mathcal{V}}^{2}\right)
$$

holds for any $T>0$, then system (12) is $L^{2}$ null-controllable at any time.

Remark 2.2. The assumptions of Theorem 2.1 may seem strange. Indeed, they naturally appear when we apply to the abstract system the same ideas which allowed us to treat the example in the previous section. Let us briefly explain these ideas. If we had to control system (12) by two functions (one for each equation of the system), we should have to prove the estimate:

$$
|u(T)|_{\mathcal{U}}^{2}+|v(T)|_{\mathcal{V}}^{2} \leq c_{T}\left(\int_{0}^{T}|u(t)|_{\mathcal{V}}^{2} \mathrm{~d} t+\int_{0}^{T}|v(t)|_{\mathcal{V}}^{2} \mathrm{~d} t\right),
$$

for the solution $(u, v)$ of the adjoint system (14). If this estimate is obtained, the difficulty now is to get rid of $\int_{0}^{T}|u(t)|_{\mathcal{V}}^{2} \mathrm{~d} t$. Roughly speaking, it is the role of the second equation to allow this: thus we have to find a suitable multiplier (the operator $M$ of the theorem) leading to an estimate of $\int_{0}^{T}|u(t)|_{\mathcal{V}}^{2} \mathrm{~d} t$. If we multiply the second equation of the adjoint system (14) by $M^{*} u$, we are naturally led to the assumption (18) and the other assumptions are here to manage the "bad" terms appearing after this. For instance, if $D$ was invertible (with a bounded inverse) and of the same "order" as $B$, the natural choice is $M=D^{-1}$ and $p=0$ (it corresponds to the situation in the example treated in the previous section). Based on these ideas, the functional we introduce allows both to prove (24) and to get rid of $\int_{0}^{T}|u(t)|_{\mathcal{V}}^{2} \mathrm{~d} t$ but, in doing this, the condition (23) (it is a kind of parabolic property) becomes necessary to end the proof.

Remark 2.3. For another application of this process, the reader should refer to the third section (system (41)). Proof. For $u$ and $v$ satisfying (14), define the quadratic form $\Phi$ by

$$
\Phi(u, v)=t^{l+2}\left|A^{-p} u\right|_{\mathcal{U}}^{2}+\lambda t^{l}|v|_{\mathcal{V}}^{2}-\mu t^{l+1}(M v, u)_{\mathcal{U}},
$$

where the positive parameters $l, \lambda$, and $\mu$ will be chosen later. The first step of the proof is to show that there exists a positive constant $c$ such that

Using (14) we deduce that:

$$
\frac{\mathrm{d}}{\mathrm{d} t} \Phi(u, v) \leq c|v|_{\mathcal{V}}^{2} .
$$

$$
\begin{aligned}
\frac{\mathrm{d}}{\mathrm{d} t}\left(t^{l+2}\left|A^{-p} u\right|_{\mathcal{U}}^{2}\right)= & (l+2) t^{l+1}\left|A^{-p} u\right|_{\mathcal{U}}^{2}+2 t^{l+2}\left(A^{-2 p} u, u_{t}\right)_{\mathcal{U}} \\
= & (l+2) t^{l+1}\left|A^{-p} u\right|_{\mathcal{U}}^{2}-2 t^{l+2}\left|A^{1-p} u\right|_{\mathcal{U}}^{2} \\
& +2 t^{l+2}\left(B v, A^{-2 p} u\right)_{\mathcal{U}}
\end{aligned}
$$


and

$$
\begin{aligned}
\frac{\mathrm{d}}{\mathrm{d} t}\left(t^{l}|v|_{\mathcal{V}}^{2}\right) & =l t^{l-1}|v|_{\mathcal{V}}^{2}+2 t^{l}\left(v, v_{t}\right)_{\mathcal{V}} \\
& =l t^{l-1}|v|_{\mathcal{V}}^{2}-2 t^{l}|C v|_{\mathcal{V}}^{2}+2 t^{l}(D u, v)_{\mathcal{V}}
\end{aligned}
$$

as well as

$$
\begin{aligned}
\frac{\mathrm{d}}{\mathrm{d} t}\left(t^{l+1}(M v, u)_{\mathcal{U}}\right)= & (l+1) t^{l}(M v, u)_{\mathcal{U}}+t^{l+1}\left(M v_{t}, u\right)_{\mathcal{U}}+t^{l+1}\left(M v, u_{t}\right)_{\mathcal{U}} \\
= & (l+1) t^{l}(M v, u)_{\mathcal{U}}+t^{l+1}(M D u, u)_{\mathcal{U}} \\
& -t^{l+1}\left(u,\left(A^{2} M+M C^{2}\right) v\right)_{\mathcal{U}}+t^{l+1}(B v, M v)_{\mathcal{U}}
\end{aligned}
$$

Thus, differentiating $\Phi$ with respect to time, in view of (26)-(28) we obtain

$$
\frac{\mathrm{d}}{\mathrm{d} t} \Phi(u, v)=I_{1}+I_{2}+I_{3}
$$

where we have introduced the following notations

$$
\begin{aligned}
I_{1}= & (l+2) t^{l+1}\left|A^{-p} u\right|_{\mathcal{U}}^{2}-2 t^{l+2}\left|A^{1-p} u\right|_{\mathcal{U}}^{2}-\mu t^{l+1}(M D u, u)_{\mathcal{U}}, \\
= & 2 t^{l+2}\left(B v, A^{-2 p} u\right)_{\mathcal{U}}+2 \lambda t^{l}(D u, v)_{\mathcal{V}} \\
& -\mu(l+1) t^{l}(M v, u)_{\mathcal{U}}+\mu t^{l+1}\left(u,\left(A^{2} M+M C^{2}\right) v\right)_{\mathcal{U}} \\
I_{3}= & \lambda l t^{l-1}|v|_{\mathcal{V}}^{2}-2 \lambda t^{l}|C v|_{\mathcal{V}}^{2}-\mu t^{l+1}(B v, M v)_{\mathcal{U}}
\end{aligned}
$$

Now we estimate each of the three quantities $I_{1}, I_{2}$ and $I_{3}$.

- Estimate of $I_{1}$.

In view of (18), one can choose $\mu$ large enough so that

$$
\begin{aligned}
I_{1} & \leq t^{l+1}(l+2-c \mu)\left|A^{-p} u\right|_{\mathcal{U}}^{2}-2 t^{l+2}\left|A^{1-p} u\right|_{\mathcal{U}}^{2} \\
& \leq-2 t^{l+2}\left|A^{1-p} u\right|_{\mathcal{U}}^{2} .
\end{aligned}
$$

- Estimate of $I_{2}$.

Using Cauchy-Schwarz's and then Young's inequality, we have

$$
\begin{aligned}
I_{2}= & 2 t^{l+2}\left(A^{-1-p} B v, A^{1-p} u\right)_{\mathcal{U}} \\
& +2 \lambda t^{l}\left(A^{1-p} u, A^{-1+p} D^{*} v\right)_{\mathcal{U}} \\
& -\mu(l+1) t^{l}\left(A^{-1+p} M v, A^{1-p} u\right)_{\mathcal{U}} \\
& +\mu t^{l+1}\left(A^{1-p} u,\left(A^{1+p} M+A^{-1+p} M C^{2}\right) v\right)_{\mathcal{U}} \\
\leq & \frac{1}{2} t^{l+2}\left|A^{1-p} u\right|_{\mathcal{U}}^{2}+2 t^{l+2}\left|A^{-1-p} B v\right|_{\mathcal{U}}^{2} \\
& +\frac{1}{2} t^{l+2}\left|A^{1-p} u\right|_{\mathcal{U}}^{2}+2 \lambda^{2} t^{l-2}\left|A^{-1+p} D^{*} v\right|_{\mathcal{U}}^{2} \\
& +\frac{1}{2} t^{l+2}\left|A^{1-p} u\right|_{\mathcal{U}}^{2}+\frac{1}{2} \mu^{2}(l+1)^{2} t^{l-2}\left|A^{-1+p} M v\right|_{\mathcal{U}}^{2} \\
& +\frac{1}{2} t^{l+2}\left|A^{1-p} u\right|_{\mathcal{U}}^{2}+\mu^{2} t^{l}\left|A^{1+p} M v\right|_{\mathcal{U}}^{2}+\mu^{2} t^{l}\left|A^{-1+p} M C^{2} v\right|_{\mathcal{U}}^{2}
\end{aligned}
$$


and therefore

$$
\begin{aligned}
I_{2} \leq & 2 t^{l+2}\left(\left|A^{1-p} u\right|_{\mathcal{U}}^{2}+\left|A^{-1-p} B v\right|_{\mathcal{U}}^{2}\right) \\
& +\mu^{2} t^{l}\left(\left|A^{1+p} M v\right|_{\mathcal{U}}^{2}+\left|A^{-1+p} M C^{2} v\right|_{\mathcal{U}}^{2}\right) \\
& +2 t^{l-2}\left(\lambda^{2}\left|A^{-1+p} D^{*} v\right|_{\mathcal{U}}^{2}+\frac{1}{4} \mu^{2}(l+1)^{2}\left|A^{-1+p} M v\right|_{\mathcal{U}}^{2}\right) .
\end{aligned}
$$

Using (17) and (19)-(22), we have

$$
\begin{aligned}
I_{2} \leq & 2 t^{l+2}\left|A^{1-p} u\right|_{\mathcal{U}}^{2} \\
& +2 c\left(t^{l+2}+\mu^{2} t^{l}\right)|C v|_{\mathcal{V}}^{2} \\
& +2 t^{l-2}\left(\lambda^{2}+\frac{1}{4} \mu^{2}(l+1)^{2}\right)\left(\varepsilon|C v|_{\mathcal{V}}^{2}+c \varepsilon^{-\gamma}|v|_{\mathcal{V}}^{2}\right)
\end{aligned}
$$

or, setting

$$
\begin{aligned}
& \varepsilon=t^{2}\left(\lambda^{2}+\frac{1}{4} \mu^{2}(l+1)^{2}\right)^{-1} \text { and } l=2+2 \gamma \geq 0 \\
& I_{2} \leq \quad 2 t^{2 \gamma+4}\left|A^{1-p} u\right|_{\mathcal{U}}^{2} \\
&+\left(2 c t^{2 \gamma+4}+2 c \mu^{2} t^{2 \gamma+2}+2 t^{2 \gamma+2}\right)|C v|_{\mathcal{V}}^{2} \\
&+4\left(\lambda^{2}+\frac{1}{4} \mu^{2}(2 \gamma+3)^{2}\right)^{1+\gamma}|v|_{\mathcal{V}}^{2} .
\end{aligned}
$$

- Estimate of $I_{3}$.

To estimate $I_{3}$ we use (15) to obtain

$$
I_{3} \leq \lambda(2+2 \gamma) t^{2 \gamma+1}|v|_{\mathcal{V}}^{2}+\left(-2 \lambda t^{2 \gamma+2}+c \mu t^{2 \gamma+3}\right)|C v|_{\mathcal{V}}^{2}
$$

Now in view of (30), (32) and (33), which we substitute into (29) we have

$$
\begin{aligned}
\frac{\mathrm{d}}{\mathrm{d} t} \Phi(u, v) \leq & \left(-2 \lambda t^{2 \gamma+2}+c \mu t^{2 \gamma+3}+2 c t^{2 \gamma+4}+2 c \mu^{2} t^{2 \gamma+2}+2 t^{2 \gamma+2}\right)|C v|_{\mathcal{V}}^{2} \\
& +\left(4\left(\lambda^{2}+\frac{1}{4} \mu^{2}(2 \gamma+3)^{2}\right)^{1+\gamma}+\lambda(2+2 \gamma) t^{2 \gamma+1}\right)|v|_{\mathcal{V}}^{2} .
\end{aligned}
$$

Going back to (25), recalling (16) and making $\lambda$ larger if necessary, we remark that

$$
\Phi(u, v) \geq \frac{1}{2}\left(t^{2 \gamma+4}\left|A^{-p} u\right|_{\mathcal{U}}^{2}+t^{2 \gamma+2}|v|_{\mathcal{V}}^{2}\right) .
$$

Thus integration of (34) over [0,T/2] yields:

$$
\left|A^{-p} u(T / 2)\right|_{\mathcal{U}}^{2}+|v(T / 2)|_{\mathcal{V}}^{2} \leq c(T) \int_{0}^{T / 2}|v(t)|_{\mathcal{V}}^{2} \mathrm{~d} t,
$$

with for $T$ small:

$$
c(T) \sim T^{-2 \gamma-4}
$$

which already implies the null-controllability of system (12) in the case $p \leq 0$. Otherwise use first (23) on the interval $[T / 2, T]$ and then (35) to obtain

$$
\begin{aligned}
|u(T)|_{\mathcal{U}}^{2}+|v(T)|_{\mathcal{V}}^{2} & \leq\left(1+c T^{-q}\right)\left(\left|A^{-p} u(T / 2)\right|_{\mathcal{U}}^{2}+|v(T / 2)|_{\mathcal{V}}^{2}\right) \\
& \leq c T^{-q-2 \gamma-4} \int_{0}^{T / 2}|v(t)|_{\mathcal{V}}^{2} \mathrm{~d} t \\
& \leq c T^{-q-2 \gamma-4} \int_{0}^{T}|v(t)|_{\mathcal{V}}^{2} \mathrm{~d} t .
\end{aligned}
$$




\subsection{Some necessary conditions: a counterexample}

Here, we construct an example showing that for some coupling operator, the system cannot be null-controllable.

Let $A$ be an unbounded positive definite self-adjoint operator in a Hilbert space $H$ with discrete spectrum $\left\{\lambda_{k}\right\}_{k \in \mathbb{N}}$ (definitely, $A$ could be viewed as $-\Delta$ with Dirichlet homogeneous boundary conditions). If we denote by $\left\{\phi_{k}\right\}_{k}$ the corresponding orthonormal basis of eigenfunctions, $A$ may be written

$$
A=\sum_{k} \lambda_{k}\left\langle., \phi_{k}\right\rangle \phi_{k}
$$

Therefore, if $f$ is a real function defined on $\operatorname{spec}(A)$, we set

$$
f(A):=\sum_{k} f\left(\lambda_{k}\right)\left\langle., \phi_{k}\right\rangle \phi_{k}
$$

Assume $f: \operatorname{spec}(A) \rightarrow \mathbb{R}$ satisfies

$$
0<|f(s)|<s, \quad \forall s, \quad \text { and } \quad f(s)=o(s) \quad \text { as } \quad s \rightarrow \infty,
$$

so that the operator $f(A)$ is relatively compact with respect to $A$.

Consider the following control problem: given $u_{0}, v_{0} \in H$ and a positive number $T$, find a function $h \in$ $L^{2}((0, T), H)$ such that the solution of the Cauchy problem

$$
\left\{\begin{array}{l}
u_{t}=-A u+f(A) v \\
v_{t}=-A v+f(A) u+h \\
u(0)=u_{0}, \quad v(0)=v_{0}
\end{array}\right.
$$

satisfies $u(T)=0$ and $v(T)=0$.

The desired $h$ exists if and only if there exists a positive constant $C_{T}$ such that any solution $(u, v)$ of the homogeneous problem

satisfies the estimate

$$
\left\{\begin{array}{l}
u_{t}=-A u+f(A) v \\
v_{t}=-A v+f(A) u \\
u(0)=u_{0}, \quad v(0)=v_{0}
\end{array}\right.
$$

$$
\|u(T)\|^{2}+\|v(T)\|^{2} \leq C_{T} \int_{0}^{T}\|v(t)\|^{2} \mathrm{~d} t,
$$

where we denote by $\|$.$\| the norm in H$.

The main result of this section is:

\section{Theorem 2.4.}

1/- System (36) is null-controllable in time $T$ if and only if the function $s^{-3} f^{2}(s) \mathrm{e}^{2 T s}$ stays bounded away from zero as $s \rightarrow \infty$.

2/- If $f(s)$ decays polynomially, the system is null-controllable for any $T>0$.

3/- For $f(s)=\mathrm{e}^{-s}$ we have the null-controllability result for any $T>1$, but not for smaller values of $T$.

4/- If the decay rate of $f(s)$ is super-exponential, the system is never null-controllable.

Remark 2.5. Note that if $f$ satisfies one of the condition of this theorem, then $f(A)$ is a compact operator (as a limit of finite rank operators).

From an heuristic point of view, this result gives some indications on the "order" of the coupling operators for the null-controllability property to hold. For instance, if the coupling operator is a partial differential operator of order 0 or 1 , or if it is of order $A^{-p}$ for some real $p>0$, then (may be with some additional assumptions) the 
null-controllability of the system should hold. If the coupling operator is "too compact" (by this, we mean that its sequence of eigenvalues decays very fast to 0 ), then the null-controllability property will in general be false.

Proof. In terms of quadratic forms, (37) reads as follows:

$$
\mathrm{e}^{-2 L T} \leq C_{T} \int_{0}^{T} \mathrm{e}^{-L t} P \mathrm{e}^{-L t} \mathrm{~d} t
$$

where $L$ and $P$ are operators in $H \times H$ given by

$$
L=\left(\begin{array}{cc}
A & -f(A) \\
-f(A) & A
\end{array}\right), \quad P=\left(\begin{array}{ll}
0 & 0 \\
0 & I
\end{array}\right) .
$$

It is easy to see that the eigenvalues of the $2 \times 2$ matrix $L$ are $\lambda_{ \pm}(A)$, where $\lambda_{ \pm}(s)=s \pm|f(s)|$, and that the orthogonal matrix

diagonalizes $L$ with

$$
V=\frac{1}{\sqrt{2}}\left(\begin{array}{cc}
1 & -1 \\
1 & 1
\end{array}\right)
$$

$$
\Lambda=\left(\begin{array}{cc}
\lambda_{+}(A) & 0 \\
0 & \lambda_{-}(A)
\end{array}\right)=V L V^{*} .
$$

So, it is easy to check that

$$
\mathrm{e}^{-\Lambda t}=V \mathrm{e}^{-L t} V^{*}
$$

Thus:

with

$$
\mathrm{e}^{-2 \Lambda T} \leq C_{T} \int_{0}^{T} \mathrm{e}^{-\Lambda t} V P V^{*} \mathrm{e}^{-\Lambda t} \mathrm{~d} t
$$

$$
V P V^{*}=\frac{1}{2}\left(\begin{array}{cc}
I & -I \\
-I & I
\end{array}\right) .
$$

For any real $s$ we set $\eta(s)=\left(\mathrm{e}^{s}-1\right) / s$. Computing explicitly the integral at the right-hand side of (39), one can rewrite the null-controllability condition in the form

$$
I \leq \frac{C_{T}}{2} B(A)
$$

where

$$
B(s)=\left(\begin{array}{cc}
\eta\left(2 T \lambda_{+}(s)\right) & -\eta\left(-T \lambda_{+}(s)-T \lambda_{-}(s)\right) \\
-\eta\left(-T \lambda_{+}(s)-T \lambda_{-}(s)\right) & \eta\left(2 T \lambda_{-}(s)\right)
\end{array}\right) .
$$

Denote by $\sigma(s)$ the smallest eigenvalue of the $2 \times 2$ matrix $B(s)$. Clearly, using assumptions on $f(s)$

$$
\frac{\operatorname{det} B(s)}{\operatorname{tr} B(s)} \leq \sigma(s) \leq 2 \frac{\operatorname{det} B(s)}{\operatorname{tr} B(s)}
$$

Since inequality (40) is equivalent to the positive definiteness of the operator $\sigma(A)$, we arrive at the following assertion: system (36) is null-controllable if and only if the function $\operatorname{det} B(s) / \operatorname{tr} B(s)$ is bounded away from zero.

To estimate $\operatorname{det} B(s) / \operatorname{tr} B(s)$ first note that

$$
(\ln \eta(s))^{\prime \prime}=\frac{1}{s^{2}}-\frac{1}{\mathrm{e}^{s}+\mathrm{e}^{-s}-2} .
$$


Thus the function $\eta$ is log-convex and

$$
\frac{1}{s^{2}+12} \leq(\ln \eta(s))^{\prime \prime} \leq \frac{1}{s^{2}}
$$

Applying the Taylor formula to $\ln \eta(s)$, for two real numbers $s_{1}$ and $s_{2}$ we have

$$
\eta\left(2 s_{1}\right) \eta\left(2 s_{2}\right)=\eta^{2}\left(s_{1}+s_{2}\right) \mathrm{e}^{(\ln \eta(\bar{s}))^{\prime \prime}\left(s_{1}-s_{2}\right)^{2}}
$$

where $\bar{s} \in\left[s_{1}, s_{2}\right]$. Replacing $s_{1}$ and $s_{2}$ by $\lambda_{+}(s)$ and $\lambda_{-}(s)$, respectively, we obtain the following expression for the determinant of the matrix $B(s)$ :

$$
\operatorname{det} B(s)=\eta\left(2 T \lambda_{+}(s)\right) \eta\left(2 T \lambda_{-}(s)\right)\left[1-\mathrm{e}^{-4 f^{2}(s) T^{2} \psi(s)}\right]
$$

where

$$
\frac{1}{4 T^{2} \lambda_{+}^{2}(s)+12} \leq \psi(s) \leq \frac{1}{4 T^{2} \lambda_{-}^{2}(s)}
$$

This, in particular, shows that the function $\operatorname{det} B(s) / \operatorname{tr} B(s)$ is strictly positive for any value of $s$. To verify if it is bounded away from zero it suffices to study its asymptotic behavior as $s \rightarrow \infty$. As is easy to see,

$$
\begin{aligned}
\lambda_{ \pm}(s) & =s(1+o(1)), \\
\eta\left(2 \lambda_{ \pm}(s)\right) & =\frac{\mathrm{e}^{2 T s}}{2 T s}(1+o(1)), \psi(s)=\frac{1}{4 T s^{2}}(1+o(1)), \\
1-\mathrm{e}^{-4 f^{2}(s) T^{2} \psi(s)} & =\frac{f^{2}(s)}{s^{2}}(1+o(1)),
\end{aligned}
$$

and therefore

$$
\operatorname{det} B(s)=\frac{f^{2}(s) \mathrm{e}^{4 T s}}{4 T^{2} s^{4}}(1+o(1)), \quad \operatorname{tr} B(s)=\frac{\mathrm{e}^{2 T s}}{T s}(1+o(1)) .
$$

Finally we obtain

$$
\frac{\operatorname{det} B(s)}{\operatorname{tr} B(s)}=\frac{f^{2}(s) \mathrm{e}^{2 T s}}{4 T s^{3}}(1+o(1))
$$

\section{Application to the NUll-CONTROLlability of POWER-Like Systems}

\subsection{Preliminaries}

Let $\Omega$ be a bounded domain in $R^{n}$ with $C^{\infty}$ boundary and let $\omega$ be any subdomain of $\Omega$ with characteristic function $\chi_{\omega}$. For $T>0$, we set $Q_{T}=(0, T) \times \Omega$ and we consider the system of parabolic equations:

$$
\left\{\begin{array}{lll}
\varphi_{t}=-L \varphi+L^{\alpha} w & \text { in } & (0, T) \times \Omega \\
w_{t}=L^{\alpha} \varphi-L^{\beta} w+\chi_{\omega} u & \text { in } & (0, T) \times \Omega \\
\varphi(0)=\varphi_{0}, w(0)=w_{0} & \text { in } & \Omega,
\end{array}\right.
$$

where $L$ is the realization of the Dirichlet Laplacian operator on $\Omega$ :

$$
L=-\Delta, D(L)=H^{2}(\Omega) \cap H_{0}^{1}(\Omega),
$$


$\alpha \in \mathbb{R}, \beta \in \mathbb{R}^{+*}, \varphi_{0}, w_{0} \in L^{2}(\Omega)$ and $u \in L^{2}\left(Q_{T}\right)$. We then set $H=L^{2}(\Omega) \times L^{2}(\Omega)$ and we define the linear and unbounded operator:

$$
\mathcal{L}=\left(\begin{array}{cc}
L & -L^{\alpha} \\
-L^{\alpha} & L^{\beta}
\end{array}\right)
$$

with domain $D(\mathcal{L})=\left(D(L) \cap D\left(L^{\alpha}\right)\right) \times\left(D\left(L^{\alpha}\right) \cap D\left(L^{\beta}\right)\right)$.

We also denote by $<., .>$ and $\|$.$\| the scalar product and associated norm in H$ and by (.,.) and |.| the scalar product and associated norm in $L^{2}(\Omega)$. Introducing $\left(\mu_{k}\right)_{k \in \mathbb{N}^{*}}$ and $\left(\varphi_{k}\right)_{k \in \mathbb{N}^{*}}$ the sequences of eigenvalues and normalized eigenfunctions of $L$, the eigenvalues and corresponding normalized (in $H$ ) eigenfunctions of $\mathcal{L}$ are given by:

$$
\begin{aligned}
\lambda_{k}^{ \pm} & =\frac{\mu_{k}+\mu_{k}^{\beta} \mp \sqrt{\left(\mu_{k}-\mu_{k}^{\beta}\right)^{2}+4 \mu_{k}^{2 \alpha}}}{2} \\
\Phi_{k}^{ \pm} & =\frac{1}{\sqrt{\mu_{k}^{2 \alpha}+\left(-\lambda_{k}^{ \pm}+\mu_{k}\right)^{2}}}\left(\begin{array}{c}
\mu_{k}^{\alpha} \\
-\lambda_{k}^{ \pm}+\mu_{k}
\end{array}\right) \varphi_{k},
\end{aligned}
$$

To simplify the notations, we set:

$$
\begin{gathered}
\lambda_{k}=\left\{\begin{array}{lc}
\lambda_{k}^{+} & \text {if } k \geq 1 \\
\lambda_{k}^{-} & \text {if } k \leq-1,
\end{array}\right. \\
\Phi_{k}= \begin{cases}\Phi_{k}^{+} & \text {if } k \geq 1 \\
\Phi_{k}^{-} & \text {if } k \leq-1 .\end{cases}
\end{gathered}
$$

Clearly $\left(\Phi_{k}\right)_{k \in \mathbb{Z}^{*}}$ is an orthonormal family in $H$. Actually, it is total (i.e. $\overline{\operatorname{span}\left\{\left(\Phi_{k}\right)_{k \in \mathbb{Z}^{*}}\right\}}=H$ ).

We can now introduce the self-adjoint extension of $\mathcal{L}$ (for which we keep the same notation) with respect to its base of eigenfunctions according to:

$$
\begin{aligned}
\mathcal{L} & =\sum_{k \in \mathbb{Z}^{*}} \lambda_{k}<., \Phi_{k}>\Phi_{k} \\
D(\mathcal{L}) & =\left\{Y \in H, \sum_{k \in \mathbb{Z}^{*}} \lambda_{k}^{2}\left\|Y_{k}\right\|^{2}<\infty\right\} .
\end{aligned}
$$

where $Y_{k}:=<Y, \Phi_{k}>$. A necessary and sufficient condition for $-\mathcal{L}$ to generate a $C^{0}$-semigroup $\left(\mathrm{e}^{-\mathcal{L} t}\right)$ is then that:

$$
\exists C>0, \quad-\lambda_{k} \leq C, \forall k \in \mathbb{Z}^{*},
$$

and this last condition is, from the expression of $\lambda_{k}^{+}$(see (42)), equivalent to:

$$
2 \alpha \leq \beta+1 \text {. }
$$

Together with $\mathcal{L}$ defined by (44)-(45), we introduce the control operator $B: L^{2}(\Omega) \rightarrow H$ by setting

$$
B u=\left(\begin{array}{c}
0 \\
\chi_{\omega} u
\end{array}\right) .
$$

Using the notation $Y_{0}=\left(\varphi_{0}, w_{0}\right)$, system (41) can be rewritten as:

$$
\left\{\begin{aligned}
Y^{\prime} & =-\mathcal{L} Y+B u \\
Y(0) & =Y_{0} \in H
\end{aligned}\right.
$$


We will now prove the null-controllability of (47) namely that, for any $Y_{0} \in H$, there exists a control $u \in L^{2}\left(Q_{T}\right)$ such that the solution $Y$ of $(47)$ satisfies $Y(T)=0$. Noting that $B^{*}=\left(0, \chi_{\omega}\right)$, this property amounts to prove that any solution $Y=\left(\begin{array}{c}\varphi \\ w\end{array}\right)$ of the adjoint problem

$$
\left\{\begin{aligned}
Y^{\prime} & =-\mathcal{L} Y \\
Y(0) & =Y_{0}
\end{aligned}\right.
$$

satisfies the estimate:

$$
|\varphi(T)|^{2}+|w(T)|^{2} \leq C_{T} \int_{0}^{T} \int_{\omega}|w(t, x)|^{2} \mathrm{~d} x \mathrm{~d} t,
$$

For a single parabolic equation, this property is well-known and has been obtained in particular by Lebeau-Robbiano [6] and Fursikov-Imanuvilov [7]. In our case two more difficulties occur. The first one is due to the fact that the control force only acts on a single equation of this system and the second one is that this control force is localized in a subdomain of $\Omega$.

To prove (49), we will find in a first step the set of $\alpha$ and $\beta$ for which this inequality is valid if $\omega=\Omega$. This result is obtained as a direct consequence of Theorem 2.1 in the particular case of system (47). We will restrict ourselves to the case

$$
2 \alpha<\beta+1 .
$$

For these values of $\alpha$ and $\beta$, we then adapt the method developed in [6] to prove (49).

\subsection{Null controllability when $\omega=\Omega$}

The main result of this section is:

Theorem 3.1. Assume that $\beta>0$ and $2 \alpha<\beta+1$. Then for all $\varphi_{0}, w_{0} \in L^{2}(\Omega)$ and all time $T>0$ there exists a constant $C_{T}>0$ such that the corresponding solution of (48) satisfies

$$
|\varphi(T)|^{2}+|w(T)|^{2} \leq C_{T} \int_{0}^{T} \int_{\Omega}|w(t, x)|^{2} \mathrm{~d} x \mathrm{~d} t .
$$

Proof. We prove Theorem 3.1 by showing that the assumptions (15)-(23) of Theorem 2.1 hold.

We first concentrate on (15)-(22). Therefore we have to find $p \in \mathbb{R}$ and $M: L^{2}(\Omega) \rightarrow L^{2}(\Omega)$ such that $D\left(M^{*}\right) \subset D\left(L^{-\frac{p}{2}}\right)$ and such that the estimates (15)-(22) are satisfied.

We seek $M$ as a power of $L$ namely $M:=L^{-\alpha-p}$. Estimates (15) and (18) are then automatically satisfied while the estimates (16)-(22) give

$$
(S)\left\{\begin{array}{ccc}
-\alpha-p \leq \frac{-p}{2} & \text { for } & (16) \\
\frac{-1-p}{2}+\alpha \leq \frac{\beta}{2} & \text { for } & (17) \\
\frac{1+p}{2}-\alpha-p \leq \frac{\beta}{2} & \text { for } & (19) \\
\frac{-1+p}{2}-\alpha-p+\beta \leq \frac{\beta}{2} & \text { for } & (20) \\
\frac{-1+p}{2}+\alpha<\frac{\beta}{2} & \text { for } & (21) \\
\frac{-1+p}{2}-\alpha-p<\frac{\beta}{2} & \text { for } & (22)
\end{array}\right.
$$

with for $(21)$

and for $(22)$

$$
\left|L^{\frac{-1+p}{2}+\alpha} w\right|^{2} \leq \varepsilon\left|L^{\frac{\beta}{2}} w\right|^{2}+\frac{c}{\varepsilon^{\gamma}}|w|^{2}
$$

$$
\left|L^{\frac{-1+p}{2}-\alpha-p} w\right|^{2} \leq \varepsilon\left|L^{\frac{\beta}{2}} w\right|^{2}+\frac{c}{\varepsilon^{\gamma}}|w|^{2} .
$$


In order to find $\gamma$, remark that the first inequality of system $(S)$ leads to

$$
\left|L^{\frac{-1+p}{2}-\alpha-p} w\right|=\left|L^{\frac{-1-p}{2}-\alpha} w\right| \leq c\left|L^{\frac{-1+p}{2}+\alpha} w\right|
$$

so that (52) implies (53). If $\frac{-1+p}{2}+\alpha \leq 0$, then (52) holds with $\gamma=0$. Otherwise, recall that (see [10] Th. 6.10, p. 73) for a positive selfadjoint operator $\Gamma$, for any $r \in(0,1)$ there exists $C>0$ such that for any $\rho>0$ and $x \in D(\Gamma)$

$$
\left|\Gamma^{r} x\right| \leq C \rho^{r}|x|+\rho^{r-1}|\Gamma x| .
$$

Setting $\varepsilon=\rho^{r-1}$, this last inequality writes:

$$
\left|\Gamma^{r} x\right| \leq \frac{C}{\varepsilon^{\frac{r}{1-r}}}|x|+\varepsilon|\Gamma x| .
$$

Applying this with $\Gamma=L^{\beta / 2}, r=\left(\frac{-1+p}{2}+\alpha\right) \frac{2}{\beta}$, we get (52) with:

$$
\begin{aligned}
\gamma & =\frac{r}{1-r} \\
& =\max \left\{0, \frac{-1+2 \alpha+p}{\beta+1-2 \alpha-p}\right\} .
\end{aligned}
$$

Finally we have to find $p \in \mathbb{R}$ such that system $(S)$ holds. This is equivalent to:

$$
\max (2 \alpha-1-\beta,|\beta-1|-2 \alpha)<p<1+\beta-2 \alpha
$$

and it is now easily seen that the assumptions $\beta>0$ and $2 \alpha<1+\beta$ imply the existence of such a real $p$. For example, one can verify that

$$
p:=\max \{0,|1-\beta|-2 \alpha\}
$$

is convenient.

Thus the assumptions (15)-(22) of Theorem 2.1 are satisfied and this implies the existence of a constant $c$ independent of $T$ such that the solution of (48) satisfies

$$
\left|L^{-\frac{p}{2}} \varphi(T)\right|^{2}+|w(T)|^{2} \leq c T^{-2 \gamma-4} \quad \int_{0}^{T}|w(\tau)|^{2} \mathrm{~d} \tau
$$

It remains to verify that Assumption (23) also holds for the solution of system (48). In this case, Assumption (23) becomes: there exists $q>0$ such that the solution of system (48) satisfies

$$
|\varphi(T)|^{2}+|w(T)|^{2} \leq c\left(1+T^{-q}\right)\left(\left|L^{\frac{-p}{2}} \varphi(0)\right|^{2}+|w(0)|^{2}\right) .
$$

This estimate is easily proved by writing down the solution of (48) in the basis $\left(\Phi_{k}\right)_{k \in \mathbb{Z}^{*}}$. Indeed, we have:

$$
Y(t)=\sum_{k \in \mathbb{Z}^{*}} \mathrm{e}^{-\lambda_{k} t}\left\langle Y_{0}, \Phi_{k}\right\rangle \Phi_{k}
$$


Thus, using the properties of the $\Phi_{k}$ and setting $Y_{0}=\left(\varphi_{0}, w_{0}\right)$

$$
\begin{aligned}
\|Y(t)\|^{2} & =\sum_{k \in \mathbb{Z}^{*}} \mathrm{e}^{-2 \lambda_{k} t}\left|\left\langle Y_{0}, \Phi_{k}\right\rangle\right|^{2} \\
& \leq C \sum_{k \in \mathbb{Z}^{*}} \frac{\mathrm{e}^{-2 \lambda_{k} t}}{\mu_{|k|}^{2 \alpha}+\left(-\lambda_{k}+\mu_{|k|}\right)^{2}}\left(\mu_{|k|}^{2 \alpha}\left|\left\langle\varphi_{0}, \varphi_{k}\right\rangle\right|^{2}+\left(-\lambda_{k}+\mu_{|k|}\right)^{2}\left|\left\langle w_{0}, \varphi_{k}\right\rangle\right|^{2}\right) \\
& \leq C \sum_{k \in \mathbb{Z}^{*}}\left[\mu_{|k|}^{2 p} \mathrm{e}^{-2 \lambda_{k} t}\left(\mu_{|k|}^{-2 p}\left|\left\langle\varphi_{0}, \varphi_{k}\right\rangle\right|^{2}\right)+\left|\left\langle w_{0}, \varphi_{k}\right\rangle\right|^{2}\right] .
\end{aligned}
$$

Studying the asymptotic behavior of $\lambda_{k}$ yields that there exist constants $C>0$ and $\delta>0$ such that $\lambda_{k} \geq C \mu_{|k|}^{\delta}$ for all $k \in \mathbb{Z}^{*}$. On the other hand, it is a matter of computation to check that for all $t>0$ :

$$
\mu_{|k|}^{2 p} \mathrm{e}^{-2 C \mu_{|k|}^{\delta} t} \leq \frac{C}{t^{q}}
$$

with $q=\frac{2 p}{\delta}$ and $C$ is a constant independent of $k$ and $t$. Thus:

$$
\begin{aligned}
\|Y(t)\|^{2} & \leq \sum_{k \in \mathbb{Z}^{*}}\left(\frac{C}{t^{q}} \mu_{|k|}^{-2 p}\left|<\varphi_{0}, \varphi_{k}>\right|^{2}+\left|<w_{0}, \varphi_{k}>\right|^{2}\right) \\
& \leq C\left(1+\frac{1}{t^{q}}\right)\left(\left|L^{\frac{-p}{2}} \varphi(0)\right|^{2}+|w(0)|^{2}\right)
\end{aligned}
$$

which is the desired estimate.

Thus the conclusion of Theorem 2.1 follows:

$$
|\varphi(T)|^{2}+|w(T)|^{2} \leq C_{T} \int_{0}^{T} \int_{\Omega}|w(t, x)|^{2} \mathrm{~d} x \mathrm{~d} t,
$$

where the constant $C_{T}$ is estimated by

$\gamma$ being defined in (54).

$$
C_{T}=c T^{-2 p-2 \gamma-4}
$$

\subsection{Null controllability when $\omega \Subset \Omega$}

This part is devoted to the proof of the main result of this section.

Remark 3.2. The abstract result does not apply in this situation because the control force is constrained to be in a strict subspace of $L^{2}\left(Q_{T}\right)$ (the abstract result gives a control function in the whole space without more precision).

Theorem 3.3. Assume that $\beta>0$ and $2 \alpha<\beta+1$. Then for any $Y_{0} \in H$ and any $T>0$, there exists a control $u \in L^{2}\left(Q_{T}\right)$ such that the solution $Y$ of $(47)$ satisfies $Y(T)=0$.

Proof. We give it for completeness because it is essentially the same than the one given by G. Lebeau and L. Robbiano [6] in the case of a single equation. Indeed, the original point here is Lemma 3.4 which itself uses the results of the previous section. Following [6], we fix $\delta \in(0, T / 2)$ and $\rho \in(0, b / n)$ where $b=2 \min (1, \beta)-1$ and $n$ is the space dimension. For $l \geq 1$ we set

$$
\begin{aligned}
\sigma_{l} & =2^{l}, \quad T_{l}=A 2^{-\rho l} \\
A & >0 \text { is chosen such that } 2 \sum_{l \geq 1} T_{l}=T-2 \delta .
\end{aligned}
$$


Then we introduce, for any $t>0$, the operator:

$$
L_{t}\left(Y_{0}, u\right)=\mathrm{e}^{-t \mathcal{L}} Y_{0}+\int_{0}^{t} \mathrm{e}^{-(t-s) \mathcal{L}} B u(s) \mathrm{d} s,
$$

(so $Y(t)=L_{t}\left(Y_{0}, u\right)$ is the unique solution of $(47)$ ).

We define the sequence $\left(a_{l}\right)_{l \geq 0}$ by setting

$$
\begin{aligned}
& a_{0}=\delta \\
& a_{l}=a_{l-1}+2 T_{l}, \quad l \geq 1,
\end{aligned}
$$

and for any initial data $Y_{0} \in H$ let $u_{l}=K_{T, \sigma_{l}}\left(Y_{0}\right)$ be a control such that:

$$
\pi_{\sigma_{l}}\left(L_{T}\left(Y_{0}, u_{l}\right)\right)=0,
$$

where $\pi_{\sigma_{l}}$ is the projection operator on the subspace $H_{\sigma_{l}}=\operatorname{span}\left\{\Phi_{k}^{ \pm}, 1 \leq k \leq \sigma_{l}\right\}$ of $H$. Of course, the control $u_{l}$ needs not to be unique but we will see below that, first it exists and second that it can be chosen as the one which minimizes a given cost functional.

We are now in position to construct a sequence of states:

$$
\begin{aligned}
Y_{0} & \in H \\
Y_{1} & =\mathrm{e}^{-\delta \mathcal{L} Y_{0}} \\
Z_{l} & =L_{T_{l}}\left(Y_{l}, K_{T_{l}, \sigma_{l}}\left(Y_{l}\right)\right) \\
Y_{l+1} & =\mathrm{e}^{-T_{l} \mathcal{L} Z_{l} .}
\end{aligned}
$$

We first explain briefly this process: until time $\delta=a_{0}$ the system is freely evolving and dissipates without control $\left(u=0\right.$ in (47)). On the time interval $\left(a_{0}, a_{0}+2 T_{1}\right)$, we first introduce a control $u_{1}$ which will drive $Y_{1}$ to a function of $H_{\sigma_{1}}^{\perp}$ in time $T_{1}$ and we then let again system (47) evolves freely (with $u=0$ ) from $t=a_{0}+T_{1}$ to $t=a_{0}+2 T_{1}$. We obtain the sequence $\left(Y_{l}\right)$ by repeating this construction on $\left(a_{l-1}, a_{l}\right)$. The claim of the Theorem holds true if we are able to prove that $\lim _{l \rightarrow \infty} Y_{l}=0$.

Of course, this construction needs to be justified by proving that, at any step, the control $u_{l}=K_{T_{l}, \sigma_{l}}\left(Y_{l}\right)$ which drives the initial data $Y_{l}$ to a function of $H_{\sigma_{l}}^{\perp}$ in time $T_{l}$ exists. For the solution of system (47) the following result holds:

Lemma 3.4. Let $T>0$. For any $l \geq 1$ and any $Y_{0} \in H$, there exists at least a control $u=u_{T, l}\left(., Y_{0}\right) \in$ $L^{2}(0, T ; U)$ such that

$$
\pi_{l}(Y(T))=\pi_{l}\left(L_{T}\left(Y_{0}, u\right)\right)=0
$$

where $\pi_{l}$ is the projection operator on $H_{l}=\operatorname{span}\left\{\Phi_{k}^{ \pm}, 1 \leq k \leq l\right\}$.

This control can be chosen such that:

$$
\|u\|_{L^{2}(0, T ; U)}^{2} \leq c T^{-2 p-4-2 \gamma} \mathrm{e}^{C_{2} \sqrt{\mu_{l}}}\left\|Y_{0}\right\|^{2} .
$$

Proof of the Lemma. We fix arbitrary $l \geq 1$ and $Y_{0} \in H$. Equality (62) can be rewritten as

$$
\pi_{l} \mathrm{e}^{-T \mathcal{L}} Y_{0}+\pi_{l}\left(\int_{0}^{T} \mathrm{e}^{-(T-s) \mathcal{L}} B u(s) \mathrm{d} s\right)=0 .
$$

If we denote by $D_{T}$ the operator defined from the space $L^{2}(0, T ; U)$ in $H$ by

$$
D_{T} u=\int_{0}^{T} \mathrm{e}^{-(T-s) \mathcal{L}} B u(s) \mathrm{d} s,
$$


the first part of Lemma 3.4 is equivalent to

$$
R\left(\pi_{l} \circ \mathrm{e}^{-T \mathcal{L}}\right) \subset R\left(\pi_{l} \circ D_{T}\right)
$$

This last inclusion holds if and only if (see for instance [13] Th. 2.2, p. 208) there exists $C=C_{T, l}>0$ such that

$$
\left\|\mathrm{e}^{-T \mathcal{L}^{*}} \pi_{l}\left(Y_{0}\right)\right\|_{H} \leq C\left\|D_{T}^{*} \pi_{l}\left(Y_{0}\right)\right\|_{L^{2}(0, T ; U)} \quad \forall Y_{0} \in H
$$

Since $\left(D_{T}^{*} h\right)(t)=B^{*} \mathrm{e}^{-(T-t) \mathcal{L}} h$ for any $h \in H$ and $\mathcal{L}=\mathcal{L}^{*}$, this last inequality can be written as

$$
|\varphi(T)|^{2}+|w(T)|^{2} \leq C_{T} \int_{0}^{T} \int_{\omega}|w(t, x)|^{2} \mathrm{~d} x \mathrm{~d} t
$$

for any solution $Y=\left(\begin{array}{c}\varphi \\ w\end{array}\right)$ of System (48) with $Y_{0} \in H_{l}$. So we now prove (66). From Theorem 3.1, we have, with $p$ and $\gamma$ satisfying (55) and (54):

$$
|\varphi(T)|^{2}+|w(T)|^{2} \leq c T^{-2 p-4-2 \gamma} \int_{0}^{T} \int_{\Omega}|w(t, x)|^{2} \mathrm{~d} x \mathrm{~d} t
$$

for any solution $Y=\left(\begin{array}{c}\varphi \\ w\end{array}\right)$ of System (48) with $Y_{0} \in H$. Now since $Y_{0} \in H_{l}$, we deduce from the invariance of the eigenspaces of $\mathcal{L}$ under $\mathrm{e}^{-t \mathcal{L}}, Y(t) \in H_{l}$ for any $t \in(0, T]$ and thus:

$$
Y(t, .)=\sum_{k=1}^{l}\left\{\mathrm{e}^{\lambda_{k}^{+} t}\left\langle Y_{0}, \Phi_{k}^{+}\right\rangle \Phi_{k}^{+}+\mathrm{e}^{\lambda_{k}^{-} t}\left\langle Y_{0}, \Phi_{k}^{-}\right\rangle \Phi_{k}^{-}\right\} .
$$

From this last equality and (43), we deduce that:

$$
\begin{aligned}
w(t, .) & =\sum_{k=1}^{l}\left\{\mathrm{e}^{\lambda_{k}^{+} t}\left\langle Y_{0}, \Phi_{k}^{+}\right\rangle c_{2, k}^{+}+\mathrm{e}^{\lambda_{k}^{-} t}\left\langle Y_{0}, \Phi_{k}^{-}\right\rangle c_{2, k}^{-}\right\} \varphi_{k} \\
& =\sum_{k=1}^{l} a_{k}(t) \varphi_{k},
\end{aligned}
$$

where

$$
c_{2, k}^{ \pm}=\frac{-\lambda_{k}^{ \pm}+\mu_{k}}{\sqrt{\mu_{k}^{2 \alpha}+\left(-\lambda_{k}^{ \pm}+\mu_{k}\right)^{2}}},
$$

and $\varphi_{k}$ is a normalized eigenfunction of the Dirichlet Laplacian. Now, the observability estimates on the eigenfunctions of the Laplacian due to G. Lebeau and L. Robbiano [6] assert that there exist constants $C_{1}, C_{2}>0$ such that:

or, equivalently:

$$
\sum_{k=1}^{l}\left|a_{k}(t)\right|^{2} \leq C_{1} \mathrm{e}^{C_{2} \sqrt{\mu_{l}}} \int_{\omega}\left|\sum_{k=1}^{l} a_{k}(t) \varphi_{k}(x)\right|^{2} \mathrm{~d} x
$$

$$
\int_{\Omega}|w(t, x)|^{2} \mathrm{~d} x \leq C_{1} \mathrm{e}^{C_{2} \sqrt{\mu_{l}}} \int_{\omega}|w(t, x)|^{2} \mathrm{~d} x
$$


Estimates (68) and (67) yield (66) with

$$
C_{T}=c T^{-2 p-4-2 \gamma} \mathrm{e}^{C_{2} \sqrt{\mu_{l}}} .
$$

This proves the existence of the desired control.

That $u$ can be chosen in such a way that (63) is satisfied is classical and we recall for completeness how this can be deduced from (66) with $C_{T}$ given by (69). Let $Y_{0} \in H$. For all $\varepsilon>0$, we define the quadratic convex and continuous functional $J_{\varepsilon, l}: H_{l} \rightarrow \mathbb{R}$ by

$$
J_{\varepsilon, l}\left(X_{0}\right)=\frac{1}{2} \int_{0}^{T} \int_{\omega}|v(t, x)|^{2} \mathrm{~d} x \mathrm{~d} t+\left\langle Y_{0}, X(0)\right\rangle+\varepsilon\left\|X_{0}\right\|,
$$

where $X=\left(\begin{array}{c}\psi \\ v\end{array}\right)$ is the solution of:

$$
X^{\prime}=\mathcal{L} X, \quad X(T)=X_{0} \in H_{l} .
$$

Clearly, $Z(t, x)=X(T-t, x)$ is the solution of

$$
Z^{\prime}=-\mathcal{L} Z, Z(0)=X(0)
$$

and applying (66) with $C_{T}$ given by (69) yields

$$
\begin{aligned}
\|X(0)\|^{2} & \leq C_{T} \int_{0}^{T} \int_{\omega}|v(T-t, x)|^{2} \mathrm{~d} x \mathrm{~d} t \\
& \leq C_{T} \int_{0}^{T} \int_{\omega}|v(t, x)|^{2} \mathrm{~d} x \mathrm{~d} t .
\end{aligned}
$$

This implies that for any $\delta>0$

$$
J_{\varepsilon, l}\left(X_{0}\right) \geq\left(\frac{1}{2}-\delta C_{T}\right) \int_{\omega}|v(t, x)|^{2} \mathrm{~d} x \mathrm{~d} t-C_{\delta}\left\|Y_{0}\right\|^{2}+\varepsilon\left\|X_{0}\right\|,
$$

and then for sufficiently small values of $\delta$ we obtain

$$
J_{\varepsilon, l}\left(X_{0}\right) \geq \varepsilon\left\|X_{0}\right\|-C_{\delta}\left\|Y_{0}\right\|^{2} .
$$

It follows that $J_{\varepsilon, l}$ is a strictly convex, continuous and coercive functional and, thus, achieves a unique minimum at $\widehat{X}_{0, \varepsilon} \in H_{l}$. Let $\widehat{X}_{\varepsilon}=\left(\begin{array}{c}\widehat{\psi_{\varepsilon}} \\ \widehat{v_{\varepsilon}}\end{array}\right)$ be the solution of (70) associated with the initial data $\widehat{X}_{0, \varepsilon}$ and let $Y_{\varepsilon}$ the solution of (47) with $u:=\widehat{v_{\varepsilon}}$. It satisfies

$$
\left\|Y_{\varepsilon}(T)\right\|_{H_{l}} \leq \varepsilon
$$

Besides, one has:

$$
J_{\varepsilon, l}\left(\widehat{X}_{0, \varepsilon}\right) \leq J_{\varepsilon, l}(0)=0
$$


and thus

$$
\begin{aligned}
\int_{0}^{T} \int_{\omega}\left|\widehat{v}_{\varepsilon}(t, x)\right|^{2} \mathrm{~d} x \mathrm{~d} t & \leq-2\left\langle Y_{0}, \widehat{X}_{0, \varepsilon}\right\rangle \\
& \leq 2\left\|Y_{0}\right\|\left\|\widehat{X}_{0, \varepsilon}\right\| \\
& \leq 2 \sqrt{C_{T}}\left(\int_{0}^{T} \int_{\omega}\left|\widehat{v}_{\varepsilon}(t, x)\right|^{2} \mathrm{~d} x \mathrm{~d} t\right)^{1 / 2}\left\|Y_{0}\right\|
\end{aligned}
$$

From this last estimate, we deduces the boundedness of $\left\{\widehat{v}_{\varepsilon}\right\}_{\varepsilon>0}$ in $L^{2}([0, T] \times \omega)$. This allows us to let $\varepsilon$ going to 0 in (47) with $u:=\widehat{v_{\varepsilon}}$. From $(71)$ we deduce that the weak limit $Y$ of at least a subsequence of $\left\{Y_{\varepsilon}\right\}_{\varepsilon>0}$ satisfies

$$
\pi_{l} Y(T)=0
$$

and from the previous estimate, that the weak limit $u$ of a subsequence of $\left\{\widehat{v}_{\varepsilon}\right\}_{\varepsilon>0}$ satisfies (63). This ends the proof of the lemma.

We now come back to the proof of Theorem 3.3. From (61), since $Z_{l} \in H_{\sigma_{l}}^{\perp}$ we get

$$
\begin{aligned}
\left\|Y_{l+1}\right\| & =\left\|\mathrm{e}^{-T_{l} \mathcal{L}} Z_{l}\right\| \\
& =\left\|\sum_{k \geq \sigma_{l}+1}\left\{\mathrm{e}^{\lambda_{k}^{+} T_{l}}\left\langle Z_{l}, \Phi_{k}^{+}\right\rangle \Phi_{k}^{+}+\mathrm{e}^{\lambda_{k}^{-} T_{l}}\left\langle Z_{l}, \Phi_{k}^{-}\right\rangle \Phi_{k}^{-}\right\}\right\| \\
& =\left(\sum_{k \geq \sigma_{l}+1} \mathrm{e}^{2 \lambda_{k}^{+} T_{l}}\left|\left\langle Z_{l}, \Phi_{k}^{+}\right\rangle\right|^{2}+\mathrm{e}^{2 \lambda_{k}^{-} T_{l}}\left|\left\langle Z_{l}, \Phi_{k}^{-}\right\rangle\right|^{2}\right)^{1 / 2} \\
& \leq \mathrm{e}^{\lambda_{\sigma_{l}+1}^{+} T_{l}}\left\|Z_{l}\right\| .
\end{aligned}
$$

From (60), using that $\mathrm{e}^{-\mathcal{L} t}$ is a semigroup of contractions together with estimate (63), we obtain that:

$$
\begin{aligned}
\left\|Z_{l}\right\| & =\left\|L_{T_{l}}\left(Y_{l}, K_{T_{l}, \sigma_{l}}\left(Y_{l}\right)\right)\right\| \\
& \leq\left\|\mathrm{e}^{-T_{l} \mathcal{L}} Y_{l}\right\|+\left\|\int_{a_{l-1}}^{a_{l-1}+T_{l}} \mathrm{e}^{-\left(T_{l}-s\right) \mathcal{L}} B u_{l}(s) \mathrm{d} s\right\| \\
& \leq\left\|Y_{l}\right\|+\left\|u_{l}\right\|_{L^{2}\left(\left(a_{l-1}, a_{l-1}+T_{l}\right) \times \omega\right)} \\
& \leq\left\|Y_{l}\right\|+\sqrt{c T_{l}^{-2 p-4-2 \gamma}} \mathrm{e}^{C_{2} \sqrt{\mu_{\sigma_{l}}}}\left\|Y_{l}\right\| \\
& \leq\left(1+\sqrt{c T_{l}^{-2 p-4-2 \gamma}} \mathrm{e}^{C_{2} \sqrt{\mu_{\sigma_{l}}}}\right)\left\|Y_{l}\right\|
\end{aligned}
$$

Combining (72) and (73) yields

$$
\left\|Y_{l+1}\right\| \leq \mathrm{e}^{\lambda_{\sigma_{l}+1}^{+} T_{l}}\left(1+\sqrt{c T_{l}^{-2 p-4-2 \gamma}} \mathrm{e}^{C_{2} \sqrt{\mu_{\sigma_{l}}}}\right)\left\|Y_{l}\right\|
$$


Now, recall that $\sigma_{l}=2^{l}, T_{l}=A 2^{-\rho l}=A \sigma_{l}^{-\rho}$ and that from Weyl's formula, $\mu_{\sigma_{l}} \sim C(\Omega)\left(\sigma_{l}\right)^{2 / n}$. On the other hand, direct computations yield

with $\theta=\min (1, \beta)$. Thus

$$
\lambda_{\sigma_{l}+1}^{+} \sim-2 \mu_{\sigma_{l}+1}^{\theta} \sim-2 C \sigma_{l}^{2 \theta / n},
$$

$$
\lambda_{\sigma_{l}+1}^{+} T_{l} \sim-2 C \sigma_{l}^{\frac{2 \theta}{n}-\rho} .
$$

For simplicity we introduce the notation

$$
-a:=\frac{-2 p-4-2 \gamma}{2}
$$

Then $a>0$ and one has:

$$
\frac{C_{2} \sqrt{\mu_{\sigma_{l}}}}{\lambda_{\sigma_{l}+1}^{+} T_{l}} \sim-C \frac{\sigma_{l}^{1 / n}}{\sigma_{l}^{\frac{2 \theta}{n}-\rho}}=-C \frac{1}{\sigma_{l}^{\frac{2 \theta-1}{n}-\rho}} .
$$

Since by assumption $\rho \in\left(0, \frac{2 \theta-1}{n}\right)$, it appears that

$$
\frac{C}{T_{l}^{a}} \mathrm{e}^{\lambda_{\sigma_{l}+1}^{+} T_{l}+C_{2} \sqrt{\mu_{\sigma_{l}}}} \leq C \sigma_{l}^{a \rho} \mathrm{e}^{-C \sigma_{l}^{\frac{2 \theta}{n}-\rho}} .
$$

Coming back to (74), we get

$$
\begin{aligned}
\left\|Y_{l+1}\right\| & \leq C \sigma_{l}^{a \rho} \mathrm{e}^{-C \sigma_{l}^{\frac{2 \theta}{n}-\rho}}\left\|Y_{l}\right\| \\
& \leq C^{l} 2^{\frac{a \rho l(l+1)}{2}} \mathrm{e}^{-C 2\left(\frac{2 \theta}{n}-\rho\right) l}\left\|Y_{1}\right\| \\
& \leq C^{l} 2^{\frac{a \rho l(l+1)}{2}} \mathrm{e}^{-C 2} 2^{\left(\frac{2 \theta}{n}-\rho\right) l}\left\|Y_{0}\right\|,
\end{aligned}
$$

which shows that

$$
\lim _{l \rightarrow \infty}\left\|Y_{l+1}\right\|=0
$$

and Theorem 3.3 is proved.

\section{REFERENCES}

[1] F. Ammar-Khodja, A. Benabdallah, C. Dupaix and I. Kostine, Controllability to the trajectories of phase-field models by one control force. SIAM J. Control. Opt. 42 (2003) 1661-1680.

[2] F. Ammar-Khodja, A. Benabdallah and C. Dupaix, Controllability of some reaction-diffusion models by one control force. To appear.

[3] S. Anita and V. Barbu, Local exact controllability of a reaction-diffusion system. Diff. Integral Equ. 14 (2001) 577-587.

[4] V. Barbu, Exact controllability of the superlinear heat equation. Appl. Math. Optim. 42 (2000) 73-89.

[5] V. Barbu, Local controllability of the phase field system. Nonlinear Analysis 50 (2002) 363-372.

[6] G. Lebeau and L. Robbiano, Contrôle exact de l'équation de la chaleur. Comm. Partial Diff. Equ. 20 (1995) 335-356.

[7] A. Fursikov and O. Yu. Imanuvilov, Controllability of Evolution Equations. Seoul National University, Korea. Lect. Notes Ser. 34 (1996).

[8] E. Fernández-Cara and E. Zuazua, Null and approximate controllability for weakly blowing up semilinear heat equations. Ann. Inst. H. Poincaré, Anal. Non Linéaire 17 (2000) 583-616.

[9] O.A. Ladyženskaja, V.A. Solonnikov and N.N. Ural'ceva, Linear and Quasilinear Equations of Parabolic Type. Translations of Mathematical Monographs, AMS 23 (1968).

[10] A. Pazy, Semigroups of linear operators and applications to partial differential equations. Springer-Verlag New York (1983).

[11] T.I. Seidman, How fast are violent controls? Math. Control Signals Syst. 1 (1988) 89-95.

[12] T.I. Seidman and J. Yong, How fast are violent controls, II? Math Control Signals Syst. 9 (1997) 327-340.

[13] J. Zabczyk, Mathematical Control Theory: An Introduction. Birkhäuser (1992). 